\title{
Aspectos reproductivos del jurel Trachurus murphyi
}

\author{
Reproductive aspects of Jack mackerel Trachurus murphy
}

\author{
Ángel Perea, Julio Mori, Betsy Buitrón y Javier Sánchez
}

Instituto del Mar del Perú, esquina Gamarra y General Valle S/N Chucuito, Callao, Perú.

Email Ángel Perea: aperea@imarpe.gob.pe
Citación:

Perea A., J. Mori, B. Buitrón \& J. Sánchez. 2013. Aspectos reproductivos del jurel Trachurus murphyi en el Perú. En: Csirke J., R. Guevara-Carrasco \& M. Espino (Eds.) Ecología, pesquería y conservación del jurel (Trachurus murphyi) en el Perú. Rev. peru. biol. número especial 20(1) 029- 034 (Septiembre 2013)

\section{Resumen}

Se analiza y discute la serie temporal del Índice Gonadosomático (IGS) y la talla de primera madurez del jurel Trachurus murphyi Nichols 1920 en el Perú calculada mensualmente entre los años 1967 - 2012. Sobre la base de estos análisis se determinó la variación anual e interanual del ciclo reproductivo, además se demostró que frente a la costa del Perú T. murphyi tiene un solo periodo de desove relativamente amplio y que ocurre con un máximo en el mes de noviembre de cada año. Asimismo, se evidenció que durante más de cuatro décadas $T$. murphyi ha desovado regularmente cada año en el mar peruano. La actividad reproductiva del T. murphyi tiene una mayor variabilidad en el Perú y el periodo de desove tiene picos de menor magnitud pero de mayor amplitud con respecto al desove que ocurre frente a la costa de Chile. El análisis de las tallas de primera madurez no presentó cambios significativos a lo largo de toda la serie en el mar peruano.

Palabras clave: Reproducción, jurel, Trachurus murphyi, ciclo reproductivo, desove.

\section{Abstract}

The time series of monthly gonadosomatic index (GSI) and biannual size at first maturity of Jack mackerel Trachurus murphyi Nichols 1920 in Peru between 1967 and 2012 is analyzed and discussed. The annual and interannual variation of the reproductive cycle was determined. It is shown that in Peru T. murphyi has a single relatively extended spawning period with a maximum in November each year. It is also shown that for more than four decades T. murphyi has spawned regularly every year in Peruvian waters. The reproductive activity of $T$. murphyi has a greater variability off Peru and the spawning period has peaks of lesser magnitude but extend longer than observed in the spawning occurring off Chile. The analyses of the sizes at first maturity of Jack mackerel in Peruvian waters did not show significant changes throughout the entire period observed.

Keywords: Reproduction, Jack mackerel, Trachurus murphyi, reproductive cycle, spawning.

\section{Introducción}

Trachurus murphyi Nichols 1920 es una especie pelágica de amplia distribución en el Pacífico Sudoriental que incluye zonas de jurisdicción de Ecuador, Perú y Chile, como en alta mar. Desde el punto de vista reproductivo, se trata de un recurso con características gonocóricas (sexos separados) e iteróparos (la descendencia se produce en tandas sucesivas de desove), sin dimorfismo sexual visible. Estudios histológicos demostraron que su desarrollo ovocitario es de tipo asincrónico (Dioses et al. 1988, Gordo et al. 2008) y se determinó que esta especie tiene un patrón de desove parcial, ajustándose a la descripción de desovantes parciales de Hunter y Macewicz (1984) y de Murua y Saborido-Rey (2003).

En el Perú, Dioses et al. (1988) determinaron la talla media de madurez gonadal en $21 \mathrm{~cm}$ de longitud total (LT), verificando que T. murphyi desova con mayor intensidad entre setiembre y diciembre. Además, precisaron que durante los últimos años la principal área de desove en el Perú se ubicó entre los $14^{\circ} 00^{\prime} S$ y $18^{\circ} 30^{\prime} S$, en el frente oceánico limitado por las aguas costeras frías de intenso afloramiento y las 
aguas subtropicales superficiales, generalmente entre 100 y 150 mn de la costa.

El potencial reproductivo del jurel T. murphyi en el Perú, medido a través de la fecundidad parcial, muestra que esta especie es capaz de desovar en promedio 78789 ovocitos por tanda de desove, pudiendo alcanzar en las tallas mayores a $35 \mathrm{~cm}$ de longitud total, fecundidades parciales de hasta 162590 ovocitos por desove (Dioses et al. 1988).

En este trabajo se presentan resultados del análisis de información biológica proveniente del seguimiento de la pesquería desde el ańo 1967 al 2012, lo cual ha permitido conocer la variabilidad anual e interanual del ciclo reproductivo del $T$. murphyi en la costa peruana, sus cambios a lo largo del tiempo, así como estimar la talla de primera madurez con resolución bianual y de toda la serie.

\section{Material y métodos}

Fueron analizados un total de 145466 individuos colectados entre 1967 y 2012, por los Laboratorios Costeros del IMARPE de Paita $\left(5^{\circ} \mathrm{S}\right)$, Chimbote $\left(9^{\circ} \mathrm{S}\right)$, Huacho $\left(11^{\circ} \mathrm{S}\right)$, Callao $\left(12^{\circ} \mathrm{S}\right)$, Pisco $\left(14^{\circ} \mathrm{S}\right)$ e Ilo $\left(17^{\circ} \mathrm{S}\right)$, y que forman parte del programa de seguimiento de la pesquería pelágica. Para estos análisis se siguió la metodología descrita por Bouchon et al. (2002) que tomaron como referencia lo descrito por Holden y Raitt (1975), y se consideraron los siguientes datos: longitud total (LT), peso total, peso eviscerado y peso de la gónada. Para conocer los periodos de máxima madurez y desove se utilizó el valor promedio mensual del Índice Gonadosomático (IGS), el que se calculó como:

$I G S=100^{*}(\mathrm{Wg} / \mathrm{We})$

Donde:

$W g$ = Peso de la gónada y $W e=$ Peso eviscerado.

Para los cálculos de IGS se consideraron sólo hembras adultas iguales o mayores a $26 \mathrm{~cm} \mathrm{LT}$, en concordancia con la talla de primera madurez histórica estimada en este trabajo.

Con la finalidad de conocer los cambios anuales del IGS, se calcularon los valores medios por año, considerando solamente los de setiembre a diciembre (periodos de mayor actividad reproductiva), a los cuales se denominó "valor crítico". Se determinó el intervalo de confianza, integrando los valores de la desviación estándar, el número de muestra y la probabilidad al 95\%.

Para comparar el ciclo reproductivo de T. murphyi en Perú y Chile, se analizaron datos del IGS del jurel de Chile desde 2001 hasta 2012. Estos datos están disponibles en boletines publicados periódicamente por el IFOP en su página web (ésta página web se actualiza periódicamente y sólo mantiene los boletines más recientes). Con esos datos se calcularon el promedio y la desviación estándar mensuales, para construir los patrones que se presentan en la Figura 1. Al momento de imprimir esta publicación, el patrón para la región centro-sur se puede observar en IFOP (2013a), y el patrón para la zona norte en IFOP (2013b). Dada la amplia distribución de T. murphyi, para la comparación del ciclo reproductivo se consideraron tres zonas, dos para la parte chilena y una para la peruana, de la siguiente manera:

Norte de Chile (Arica - Antofagasta - Coquimbo)

Centro - sur de Chile (San Antonio - Guateca)

Perú (Paita - Ilo)
Para comparar los ciclos reproductivos entre zonas, los valores mensuales de IGS fueron estandarizados tomándose como máximo valor 1 y recalculándose los valores mensuales para cada serie. Con los nuevos valores calculados se obtuvieron los ciclos reproductivos para cada zona.

La talla de primera madurez fue estimada para toda la serie histórica (1967 - 2012) agrupando los datos cada dos años para tener un tamańo de muestra representativo debido a la intermitencia de la actividad pesquera. Para construir las ojivas de madurez se aseguró que las muestras utilizadas provengan de periodos de desove y correspondan a un amplio rango de tallas e incluyan individuos virginales (Vazzoler 1982, Buitrón et al. 2011). Las proporciones fueron ajustadas a una curva logística de la forma:

$$
\mathrm{y}=\mathrm{a} /\left(1+\mathrm{b}^{*} \exp (-\mathrm{cx})\right)
$$

Donde:

$\mathrm{x}=$ Longitud total; $\mathrm{a}, \mathrm{b}$ y $\mathrm{c}=$ constantes, $\mathrm{y}=\mathrm{L}_{50}$ o longitud de proporción $50 \%$ o 0.5 .

\section{Resultados y discusión}

Ciclo reproductivo.- La serie histórica completa del IGS muestra que en el Perú T. murphyi tiene un periodo de maduración y desove relativamente amplio, que se extiende entre sep-
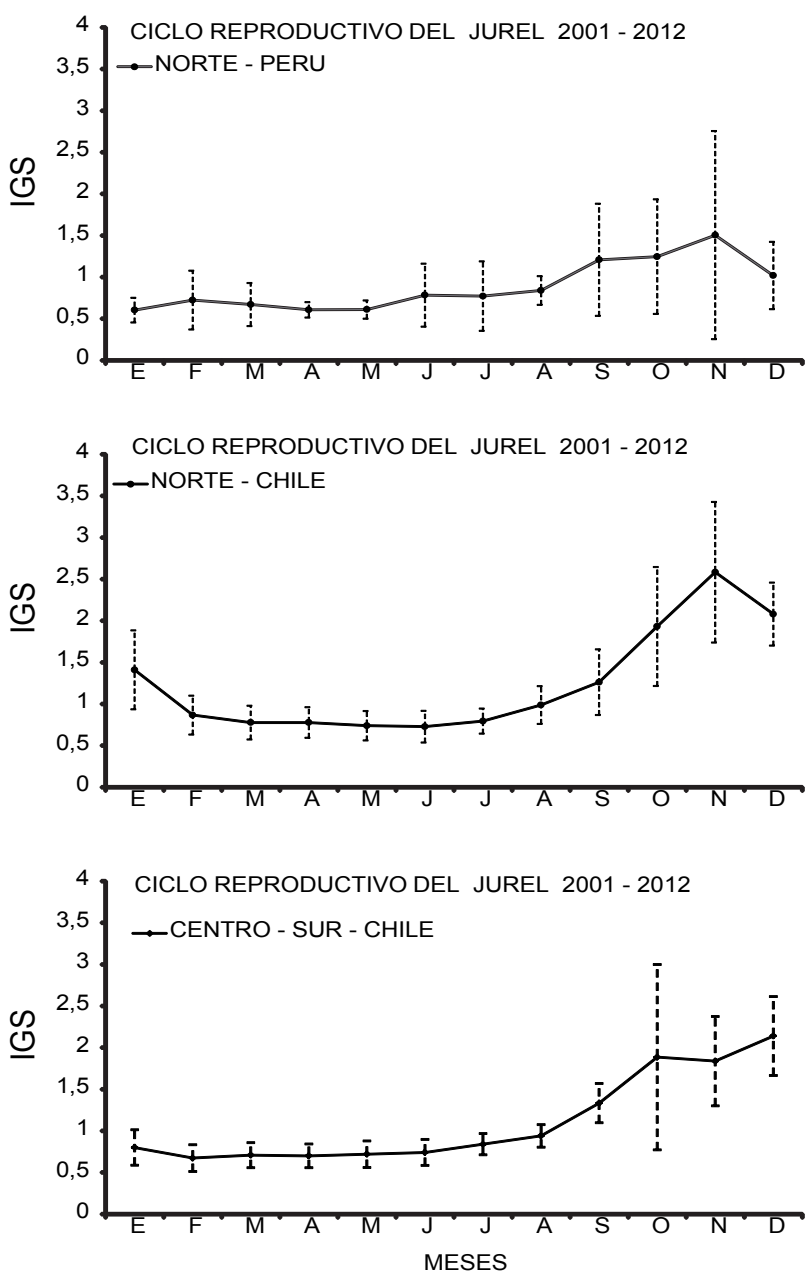

Figura 1. Ciclo reproductivo de jurel T. murphy: a) en Perú, b) al norte de Chile; y, c) al centro-sur de Chile.

Figure 1. Reproductive cycle of Jack mackerel T. murphyi: a) in Peru with information from 1962 to 2012; b) in northern Chile; and c) in southern-central Chile. 
tiembre y diciembre con máximos valores de IGS en noviembre. El patrón anual del IGS de T. murphy en Perú es claramente diferente al que se observa para el T. murphyi en el centro-sur de Chile, donde según Oyarzún et al. (1998) y Aracena et al. (1998) los valores máximos se producen en diciembre (Fig. 1).

Por otro lado, Grechina et al. (1998) afirmaron que en la subdivisión norte del Pacífico Sudoriental, T. murphyi presentaría un desove intenso entre agosto y septiembre. Sin embargo nuestros resultados muestran claramente que los máximos valores de desove de T. murphyi frente a la costa peruana se producen en noviembre.

Las diferencias temporales entre los máximos desoves que se observan T. murphyi entre la jurisdicción de Perú y Chile, también han sido observadas en otras especies pelágicas de la región, tal como la anchoveta Engraulis ringens, la cual presenta un máximo de actividad reproductiva de julio a febrero en Chile (Claramunt 2012) y de setiembre a marzo en el norte-centro de Perú (Chirinos \& Alegre 1969 , Buitron \& Perea 2000).

En peces la actividad de desove está fuertemente influenciada por las condiciones ambientales y las diferentes poblaciones y especies adaptan su calendario de desove para aprovechar ventanas de tiempo o periodos de menor incertidumbre ambiental (Wooton 1995) y una mayor probabilidad de supervivencia para sus productos de desove. Por lo tanto, la asincronía en el plazo máximo de actividad reproductiva entre el T. murphyi en Perú y el de la zona centro-sur de Chile puede ser el resultado de una respuesta diferente de estas poblaciones a los diferentes regímenes ambientales.

En la Figura 2 se muestran los valores de IGS estandarizados de T. murphyi de Perú y de la parte sur de Chile, donde se aprecian las diferencias en la amplitud y los meses que registran la máxima actividad reproductiva. Aunque no se cuenta con una serie de tiempo basada en estudios microscópicos que permita estimar la fracción desovante de T. murphyi, la característica de tener un periodo de desove más amplio con valores de IGS más bajos podría interpretarse como una estrategia reproductiva inducida por la alta variabilidad ambiental en el mar peruano, y que llevaría a T. murphyi a producir desoves menos intensos pero de mayor duración que permitirían aumentar la probabilidad de que sus estadios tempranos de vida encuentren condiciones

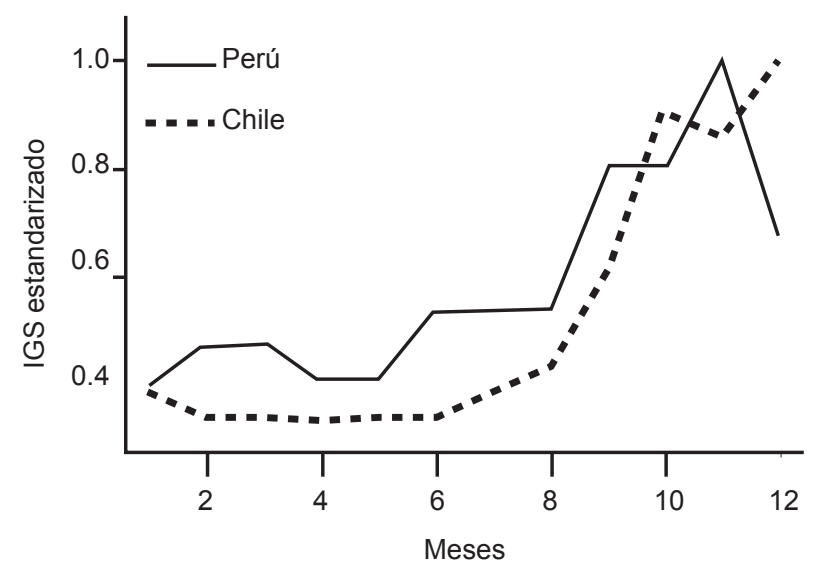

Figura 2. Ciclo reproductivo de jurel T. murphyi en Perú y sur de Chile con IGS estandarizado, años 2001-2010.

Figure 2. Reproductive cycle of Jack mackerel T. murphyi in Peru and southern Chile with standardized GSI, years 2001-2010.
Tabla 1. Principales características del ciclo reproductivo del jurel $T$. murphyi de acuerdo a la variación mensual del IGS.

Table 1. Main characteristics of the reproductive cycle of Jack mackerel $T$. murphyi according the monthly variability in the gonadosomatic index (GSI).

\begin{tabular}{cccc}
\hline & \multicolumn{3}{c}{ Características del ciclo reproductivo } \\
\cline { 2 - 4 } & $\begin{array}{c}\text { Amplitud de } \\
\text { la actividad } \\
\text { reproductiva } \\
\text { (meses) }\end{array}$ & $\begin{array}{c}\text { Mes de máxima } \\
\text { actividad } \\
\text { reproductiva } \\
\text { (Máximo valor } \\
\text { de IGS) }\end{array}$ & $\begin{array}{c}\text { Variabilidad } \\
\text { de la actividad } \\
\text { reproductiva } \\
\text { (SD) }\end{array}$ \\
\hline Perú & 4 & Noviembre & Amplia $( \pm 1.25)$ \\
Chile (norte) & 3 & Noviembre & Baja $( \pm 0.84)$ \\
Chile (centro-sur) & 3 & Diciembre & Baja $( \pm 0.54)$ \\
\hline
\end{tabular}

adecuadas para su sobrevivencia en una situación de mayor incertidumbre.

Hay tres diferencias interesantes en los ciclos reproductivos que se muestran en la Tabla 1 y que podrían ser los indicadores de diferentes adaptaciones a largo plazo en la estrategia reproductiva: (1) La amplitud del periodo máximo de desove en el Perú es de 4 meses, mientras que en el norte y en el centro-sur de Chile es de 3 meses; (2) los valores medios mensuales del IGS de T. murphyi frente a la costa peruana son inferiores a los de T. murphyi frente a la costa de Chile; y, (3) la desviación estándar del IGS en la actividad reproductiva máxima es mayor en Perú que en Chile. La mayor amplitud del periodo de desove y la desviación estándar más alta de IGS son señales claras de una respuesta biológica diferencial a un entorno más variable respecto al sur de Chile.

En general, los máximos valores de IGS son inferiores en el Perú comparados con los de T. murphyi en Chile, lo cual podría interpretarse como una menor actividad reproductiva en el mar peruano, que coincide con lo reportado por Cubillos et al. (2008). Sin embargo, en algunos años los mayores valores del IGS superan al patrón establecido, como lo registrado en los ańos 2001, 2003 y 2005 (Fig. 3).

Por otro lado, las estimaciones de abundancia de larvas de $T$. murphyi obtenidas con los cruceros de evaluación desde 1966 al 2010 (Ayón \& Correa 2013) confirman que efectivamente T. murphyi desova y recluta en el mar peruano. Además, en algunos ańos dichas estimaciones muestran altas densidades de larvas, las cuales alcanzaron valores superiores a 500 larvas $/ \mathrm{m}^{2}$ indicando desoves abundantes. Por lo tanto, y considerando que el IGS es un índice que representa el crecimiento en peso del ovario debido a los procesos de maduración y desove, el registro de valores más bajos de IGS en Perú describe un ciclo reproductivo con características distintas, que lo distingue del ciclo reproductivo del T. murphyi en Chile.

Variación interanual del IGS.- Desde el año 1967 a la fecha, la variación interanual del IGS describe tres periodos en función de valores críticos (Fig. 3): el primero, desde 1967 hasta 1985, con valor medio crítico de 1.09 ( $\mathrm{DE}=1.18$ ); el segundo, desde 1986 hasta 1998 con valor crítico de 1.54 (DE = 1.36); y el último desde 1999 hasta la actualidad, con valor crítico de $0.94(\mathrm{DE}=1.06)$. En general, el IGS ha presentado valores más altos entre 1967 y 1999, periodo en el cual la pesca de T. murphyi para uso industrial fue más alta frente a Perú 

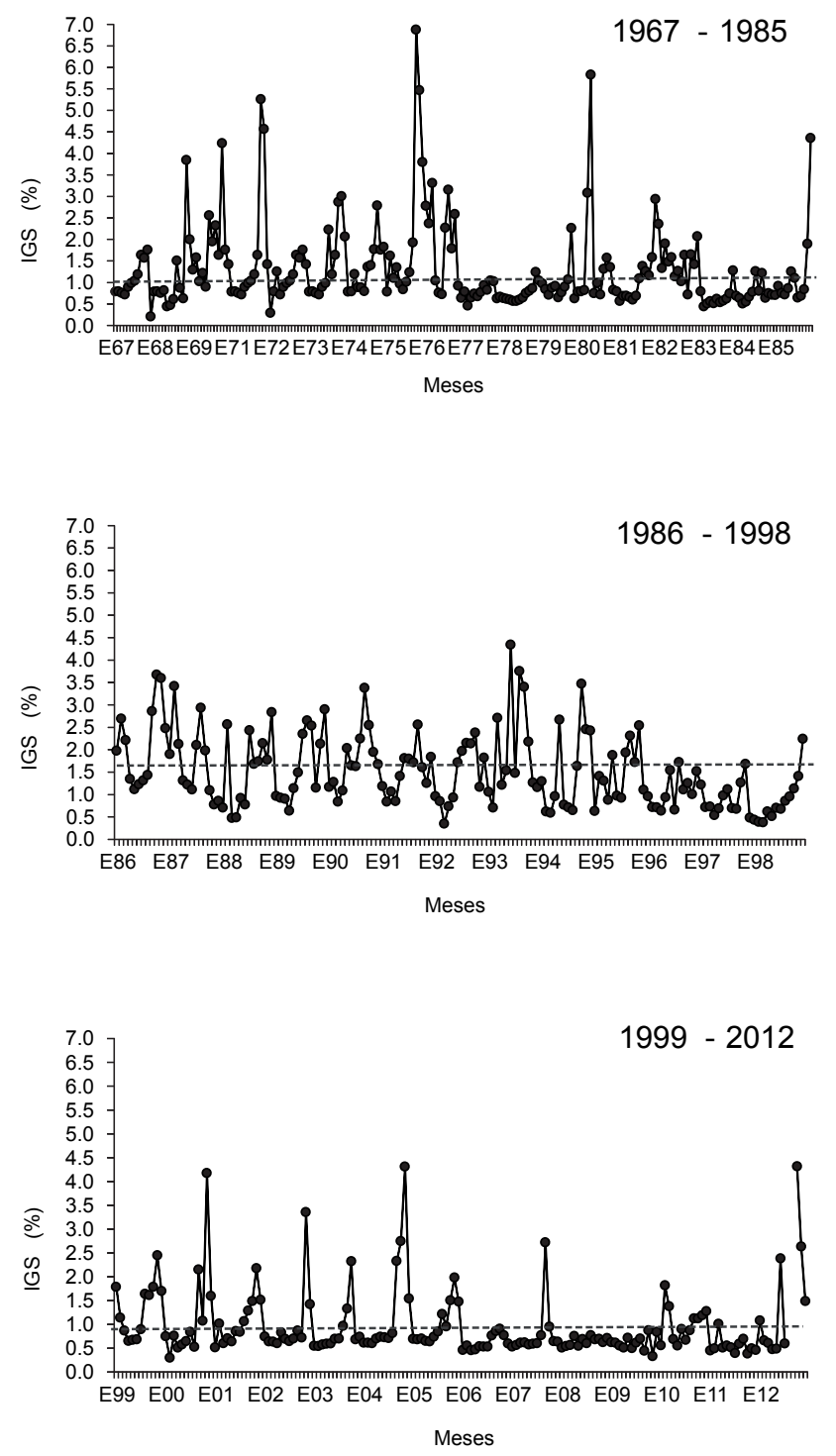

Figura 3. Tres periodos con diferentes valores críticos del IGS de jurel T. murphyi, mostrando el cambio interanual de este parámetro reproductivo.

Figure 3. Three period with different critical values of GSI of Jack mackerel T. murphyi showing interannual changes of GSI.

(Espino \& Yamashiro 2012). Sin embargo, el IGS disminuyó en la última década (2000 hasta la fecha), y durante este periodo la pesca de T. murphyi también ha declinado. Comparando los promedios de IGS de setiembre a diciembre (donde se registra la mayor actividad reproductiva del T. murphyi) para cada año, se muestra una clara tendencia negativa (Fig. 4). La que muy probablemente estaría influenciada por la mayor presencia de individuos grandes de T. murphy en la pesquería en esos ańos (1967 - 1971). Cabe anotar que en esos años la pesca de $T$. murphyi era realizada mayormente por la flota artesanal y de consumo humano directo, mientras que a mediados de los ańos 70 se incremento notablemente la pesca por parte de la flota industrial dedicada a la pesca para consumo humano indirecto y en los ańos 80 por barcos factoría. En los ańos 1967 - 71 se registraron los mayores promedios anuales de IGS con un máximo de 5.02, debido a la predominancia de ejemplares más grandes en los desembarques. En la Figura 5 se muestra el rápido aumento del IGS a partir de los $45 \mathrm{~cm}$ de longitud total que explicaría el cambio (disminución) de los valores de

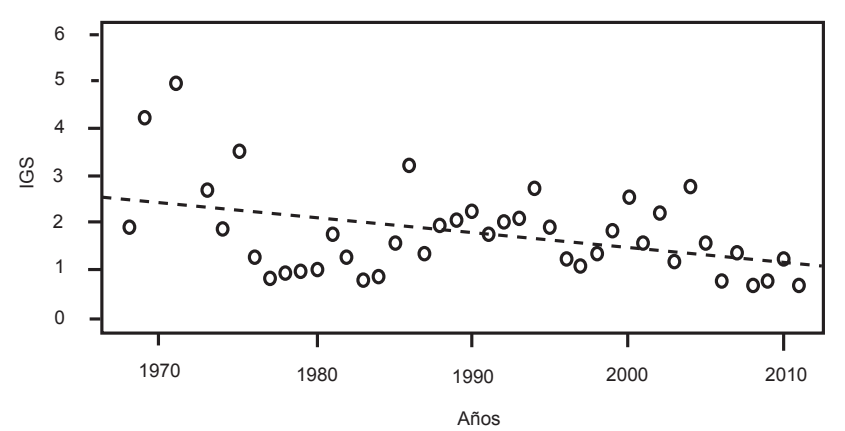

Figura 4. Media anual del IGS del jurel T. murphyi (1967-2012). Figure 4. Annual mean of Jack mackerel T. murphyi GSI (1967-2012).

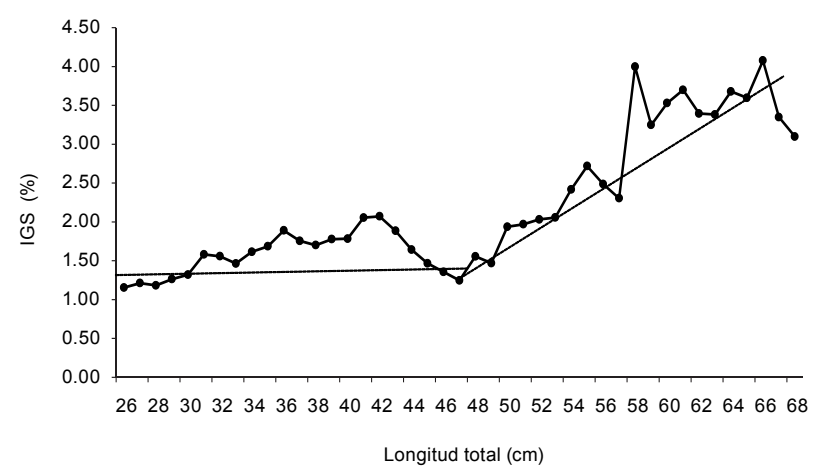

Figura 5. Índice gonadosomático de jurel T. murphyi en relación a la talla (LT).

Figure 5. Gonadosomatic index of Jack mackerel T. murphyi in relation to size $(T L)$

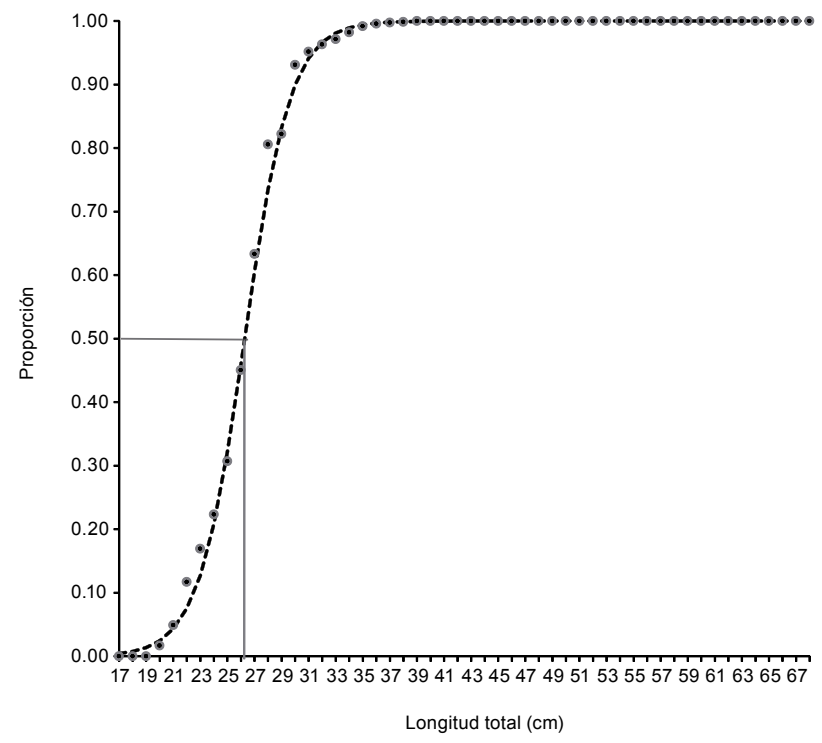

Figura 6. Ojiva de primera madurez de jurel T. murphyi frente a Perú (1967-2012).

Figure 6. Ogive of size at first maturity size Jack mackerel T. murphyi off Peru (1967-2012).

IGS promedio por la disminución de las tallas de ejemplares adultos en los desembarques a partir de los ańos 70 .

Talla de primera madurez.- La talla de primera madurez de T. murphyi entre 1967 y 2012 estimada en este estudio es 26.5 cm LT (Fig. 6). Este valor es similar a las tallas indicadas por Andrianov (1994), quien reporta que los ejemplares maduros de machos y hembras del T. murphyi comienzan a predominar en 


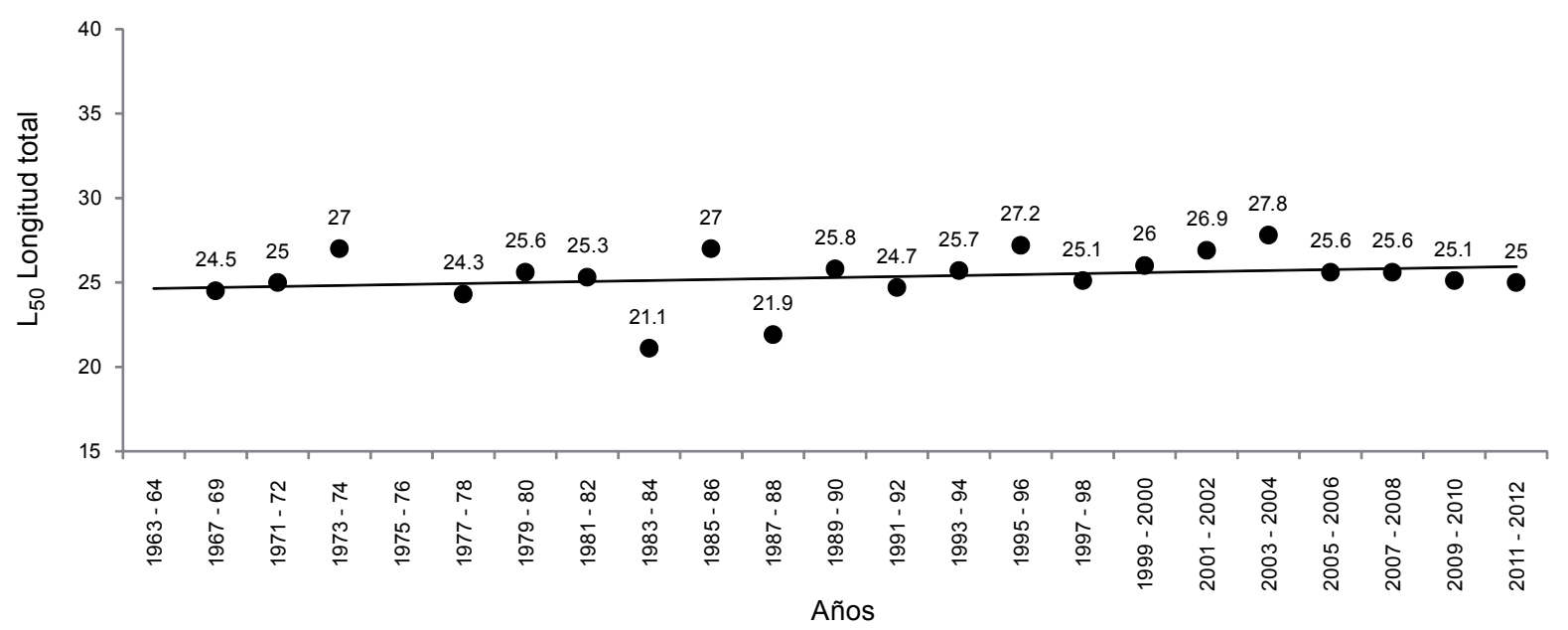

Figura 7. Tallas de primera madurez $\left(\mathrm{L}_{50}\right)$ de jurel T. murphyi para el Perú, valores bianuales de 1967 a 2012.

Figure 7. Size at first maturity $\left(L_{50}\right)$ of T. murphyi for Peru, biannual values from 1967 to 2012.

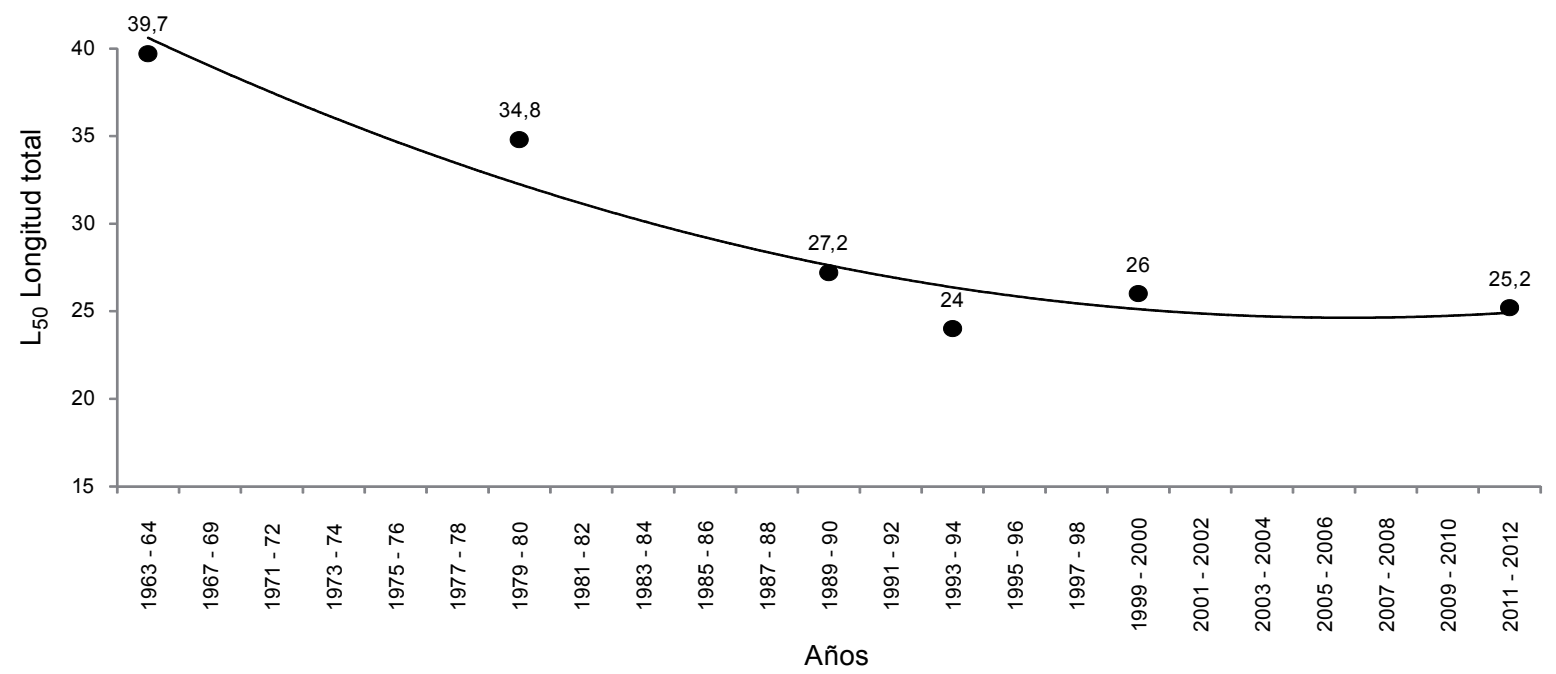

Figura 8. Tallas de primera madurez $\left(\mathrm{L}_{50}\right)$ de jurel T. murphyi para Chile, valores bianuales de 1964 a 2012.

Figure 8. Size at maturity of Jack mackerel T. murphyi for Chile, biannual values from 1964 to 2012.

tallas de 25 a $27 \mathrm{~cm}$ LT en el océano abierto de la subdivisión norte del Pacífico Sudoriental.

Las serie de estimaciones de primera madurez de T. murphyi en Perú muestran que no existe una tendencia en los valores de $\mathrm{L}_{50}$ en el periodo analizado (Fig. 7). A este respecto se destaca que en otras pesquerías se ha demostrado que la presión de pesca intensa y sostenida induce la maduración temprana de los recursos explotados (Trippel 1995). No han sido encontradas evidencias de un proceso de este tipo en la información analizada $y$, mas bien, los estimados de tallas de primera madurez de este estudio indican que no se ha producido maduración temprana alguna en el periodo observado. Se destaca que tampoco se han observado efectos sobre la talla de primera madurez relacionados con eventos tipo El Niño o con cambios de régimen.

Se hace notar que para el caso de Chile, para el mismo periodo analizado la talla de primera madurez gonadal bianual de $T$. murphyi ha venido disminuyendo de $39.7 \mathrm{~cm}$ LT en el periodo 1963 - 1964 (Kaiser 1973) a $26.0 \mathrm{~cm}$ LT en el periodo 1999 2000 (Cubillos \& Alarcón 2010), y a $25.2 \mathrm{~cm}$ LT en el periodo $2011-2012$ (Fig. 8).

\section{Literatura citada}

Andrianov D. 1994. Sobre la biología reproductiva del jurel peruano. En: Biología y pesca comercial del jurel peruano en el Pacífico Sur. Dagoberto Arcos y Alexanders Grechina (Eds). Instituto de Investigación Pesquera Talcahuano, Chile: $25-35$.

Aracena O., R. Alarcón, S. Collado, I. Lépez \& D. Arriagada. I 998. Aspectos reproductivos del jurel (Trachurus symmetricus murphyi) de la pesquería de cerco de la Octava región, Chile, 1994 - 1995. En: Biología y ecología del jurel en aguas chilenas. Instituto de Investigación Pesquera, Talcahuano-Chile, 216 pp.

Ayón P. \& J. Correa. 2013. Variabilidad espacial y temporal de larvas de jurel Trachurus murphyi en el Perú entre 1966-2010. En: Csirke J., R. Guevara-Carrasco \& M. Espino (Eds.). Ecología, pesquería y conservación del jurel (Trachurus murphyi) en el Perú. Rev. peru. biol. número especial 20(1): 009 - 020

Bouchon M., M. Niquen, J. Mori, A. Echevarría \& S. Cahuín. 2002. Manual de muestreo de la pesquería pelágica. Inf. Prog. Inst. Mar Perú Callao. 157: 38pp.

Buitrón B., A. Perea, J. Mori, J. Sánchez \& C. Roque. 2011. Protocolo para estudios de peces pelágicos y demersales. Inf. Inst. Mar Perú - Callao 38 (4): $373-384$.

Buitron B. \& A. Perea. 2000. Aspectos reproductivos de la anchoveta peruana durante el periodo 1992 - 2000. Bol Inst. Mar Perú. Bol 19 (1-2) : 45-53. 
Chirinos A. \& B. Alegre.1969. Madurez sexual de la anchoveta (Engraulis ringens J) en los periodos reproductivos 1961 - 1968. Bol. Inst. Mar Perú 2 (3).

Claramunt, G .2012. Inter-annual reproductive trait variation and spawning habitat preferences of Engraulis ringens off northern Chile. Revista de Biología Marina y Oceanografía, 47 (2): 227-243

Cubillos L.A., J. Páramo, P. Ruiz, S. Núńez \& A. Sepúlveda. 2008. The spatial structure of the oceanic spawning of Jack mackerel (Trachurus murphyi) off central Chile (1998-2001). Fisheries Research 90: 261-270. DOI: 10.1016/j.fishres.2007.10.016

Cubillos L.A. \& C. Alarcón. 2010. Estimación de la talla media de madurez sexual en Trachurus murphyi mediante parámetros del consumo relativo de oxígeno. Lat. Am. J. Aquat. Res. 38(2): 178 - 187.

Dioses T., V.H. Alarcón, M.E. Nakama \& A. Echevarría. 1988. Desarrollo ovocitario, fecundidad parcial y distribución vertical de los cardúmenes en desove del jurel Trachurus murphyi (N). En: Memorias del Simposio Internacional de los Recursos Vivos y las Pesquerías en el Pacífico Sudeste. Pp. 287 - 294.

Espino M. \& C. Yamashiro. 2012. La variabilidad climática y las pesquerías en el Pacífico Suroriental. Lat. Am. J. Aquat. Res. 40(3): 705 - 721, 2012. Latin American Journal of Aquatic Research 705. International Conference: "Environment and Resources of the South Pacific", P.M. Arana (Guest Editor). DOI: 103856/vol40 - issue3 - fulltext - 18.

Grechina A., S. Núñez \& D. Arcos. 1998. Biología reproductiva del jurel (Trachurus symmetricus murphyi) en el Pacífico Sur. En: Biología y ecología del jurel en aguas chilenas. Instituto de Investigación Pesquera, Talcahuano-Chile, 216 pp.

Gordo L., A. Costa, P. Abaunza, P. Lucio, A. Eltink \& I. Figueiredo. 2008. Determinate versus indeterminate fecundity of horse mackerel. Fisheries Research 89: 181 - 185

Holden M.J. \& D.F.S. Raitt. 1975. Manual de ciencia pesquera. Parte 2: Métodos para investigar los recursos y su aplicación. Doc. Téc. FAO Pesca (115) Rev. 1: 211 pp.

Hunter J.R. \& B.J. Macewicz. 1984. Measurement of spawning frequency in multiple spawning fishes. En: R. Lasker (ed). An egg production method for estimating spawning biomass of pelagic fish: Application to the northern anchovy (Engraulis mordax): $330 \mathrm{pp}$.
IFOP, 2013a. Informe Quincenal Nº11 (01 al 15 Junio, 2013). Asesoría Integral para la toma de decisiones en Pesca y Acuicultura, 2013. Programa de Seguimiento de las Pesquerías Pelágicas, Zona Centro - Sur 2013. Subsecretaría de Economía / Julio 2013. 08p. (www.ifop.cl)

IFOP, 2013b. Boletín Semanal N²3 (XV, I, II, III, IV Regiones) (03 al 09 Junio, 2013). Convenio I: Asesoría Integral para la Pesca y Acuicultura, 2013. Proyecto 1.10. Programa de Seguimiento de las Pesquerías Pelágicas Zona Norte 2013. Subsecretaría de Economía / Junio 2013. 15p. (www.ifop.cl)

Kaiser C. I973 Gonadal Maturation and Fecundity of Horse Mackerel Trachurus murphyi (Nichols) off the Coast of Chile. Transactions of the American Fisheries Society 102(1): $101-108$.

Murua H. \& F. Saborido-Rey. 2003. Female reproductive strategies of marine fish species of the North Atlantic. J. Northw. Atl. Fish. Sci. 33: $23-31$.

Oyarzun C., J. Chong \& M. Malagueño. 1998. Fenología reproductiva en el jurel Trachurus symetricus (Ayres 1855) (Perciformes, Carangidae) en el área Talcahuano-Chile: 1982 - 10984. En: Biología y ecología del jurel en aguas chilenas. Instituto de Investigación Pesquera, Talcahuano-Chile, $216 \mathrm{pp}$.

Trippel E. 1995. Age at maturity as a stress indicator in fisheries. BioScience 45 (11): $759-771$.

Vazzoler A. 1982. Manual de métodos para estudos biológicos de populações de peixes. Reprodução e crescimento. CNPq, Programa Nacional de Zoologia, Brasília, 2: 108p.

Wooton R.J. 1995. Ecology of teleost fishes. Published by Chapman \& Hall, $2-6$ Boundary Row, London SE1 8HN 404 pp. 